\title{
URINARY EXCRETION OF THIAMIN IN CLINICAL CASES AND THE VALUE OF SUCH ANALYSES IN THE DIAGNOSIS OF THIAMIN DEFICIENCY ${ }^{1}$
}

\author{
By WILLIAM D. ROBINSON,2 DANIEL MELNICK, ${ }^{3}$ AND HENRY FIELD, JR. \\ (From the Department of Internal Medicine, Medical School, University of Michigan, \\ Ann Arbor)
}

(Received for publication December 11, 1939)

An accurate and specific method for the determination of thiamin in urine, based on the reaction between the vitamin and diazotized p-aminoacetophenone, has been developed (1). Under suitable conditions, such determinations should give information regarding the state of saturation of an organism with this vitamin. Studies of the urinary excretion of thiamin in normal subjects before and after test doses, the factors influencing such excretion, and the changes associated with experimental deficiency have been reported (2). The present communication deals with determinations of the urinary excretion of thiamin in 89 patients in the University Hospital, correlation of these values with dietary histories of these patients, and the evaluation of the possible association of thiamin deficiency with the clinical conditions encountered.

\section{CONDITIONS AND METHODS}

The thiamin content of the diet ingested by each patient during the month prior to the test was evaluated on the basis of repeatedly consistent details of the dietary history as obtained by more than one questioner. This was classified as deficient only when obviously so, as suboptimal when the diet was low in protective foods but not grossly deficient, and as adequate when estimations indicated an intake of two-thirds of a milligram (220 international units), or more.

Two consecutive 24-hour urine specimens were collected from each patient. Just prior to the beginning of the second sample and after the largest

1 This paper was presented in part before the Central Society for Clinical Research November 3, 1939, at Chicago. The expense of this investigation was defrayed by grants from the Upjohn Company, Kalamazoo, and from the Horace H. Rackham School of Graduate Studies, University of Michigan, Ann Arbor.

2 Upjohn Fellow in Clinical Research, 1938-1940.

8 Upjohn Fellow in Clinical Research, 1937-1940. meal of the day, an aqueous solution of $5 \mathrm{mgm}$. of thiamin was taken orally. Previous studies (2) indicate the advisability of using the oral route for the test dose and the necessity of giving it with a meal. The first sample was analyzed to give the 24-hour excretion value when the diet furnished the entire supply of the vitamin. As the patients ate the same diet on the 2 consecutive days, the value for the first sample was subtracted from the value obtained by analysis of the second sample in order to calculate the percentage of the test dose excreted in the 24 hours following its administration. The method of analysis has been described in detail elsewhere (1).

Since drastic reduction of the dietary thiamin of normal subjects resulted in a rapid decrease of urinary thiamin excretion (2), the thiamin content of the diets ingested on the days of the tests is estimated in the tables. When numerical values are given, calculations are based on the tables compiled by Williams and Spies (3). Unless otherwise indicated, the tests were done before the fifth day of hospitalization. The absence of an appreciable increase in the urinary excretion during the first few days after resumption of a normal thiamin intake by a subject with experimental deficiency (2) demonstrates that the ingestion of an adequate diet by the deficient individual for a few days prior to the test does not vitiate the significance of subsequent values.

\section{RESULTS}

Standards. Standards for the interpretation of values obtained, with chief attention directed to the minimal normal excretion of thiamin, have been derived by correlating the urinary thiamin values for each subject with the adequacy of the dietary thiamin intake during the preceding month in a series of 24 normal controls (2), 22 hospital patients without clinical evidence of nutritional 


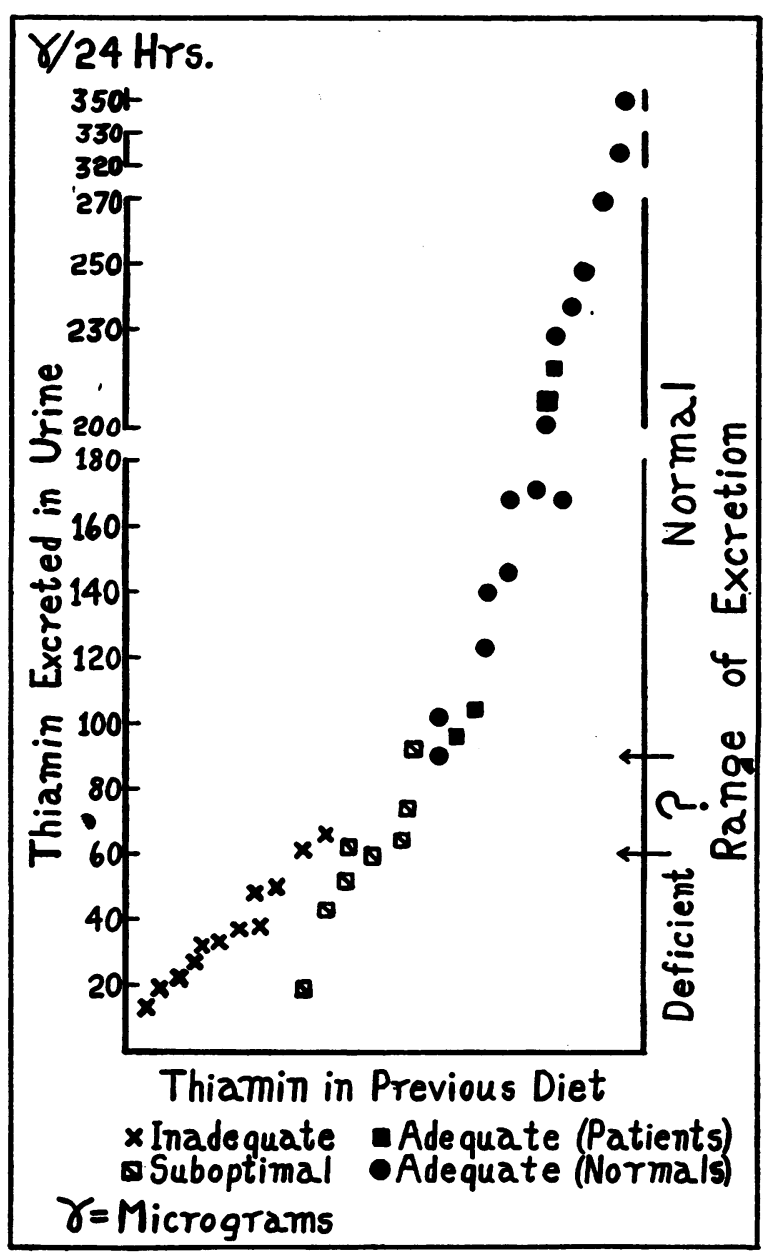

Fig. 1. Correlation in Adult Males Between the Adequacy of the Preceding Dietary Thiamin Intake and the 24-Hour Urinary Thiamin Excretion

deficiencies, and 24 patients with clinical evidence of deficiencies in one or more nutritional factors, including 3 patients with "alcoholic beriberi." All patients were excluded who had disorders which might lead to faulty absorption, storage, utilization or excretion of the vitamin.

Figure 1 presents the values of urinary thiamin excretion by male subjects during the time that the diet furnished the entire source of thiamin. Those whose preceding diets had been definitely inadequate excreted 66 micrograms or less per 24 hours, whereas all subjects who had previously been on adequate diets excreted 90 micrograms or more. With the female subjects (Figure 2) the division is not so sharp, but no subject who had taken an adequate thiamin intake excreted less than 53 micrograms per 24 hours and only 1 excreted less than 60 micrograms. Only 1 female with a history of a definitely inadequate diet excreted more than 43 micrograms. Since no significant difference was seen between males and females in the per cent of the oral dose excreted, these data for both sexes are presented in Figure 3. All patients with a history of a preceding inadequate thiamin intake excreted less than 7 per cent of the oral dose; all 27 subjects whose diets had been adequate excreted about 8 per cent or more, and 22 of the 27 excreted 10 per cent or more.

It appears that if a male subject fails to excrete more than 90 micrograms of thiamin during a 24hour period when he is ingesting an adequate diet, he may be suspected of having a significant reduction of the body stores of thiamin, and if he excretes less than 60 micrograms, such reduction is reasonably certain. Corresponding values for females place the lower limit of normal excretion at 60 micrograms, with values below 40 micrograms

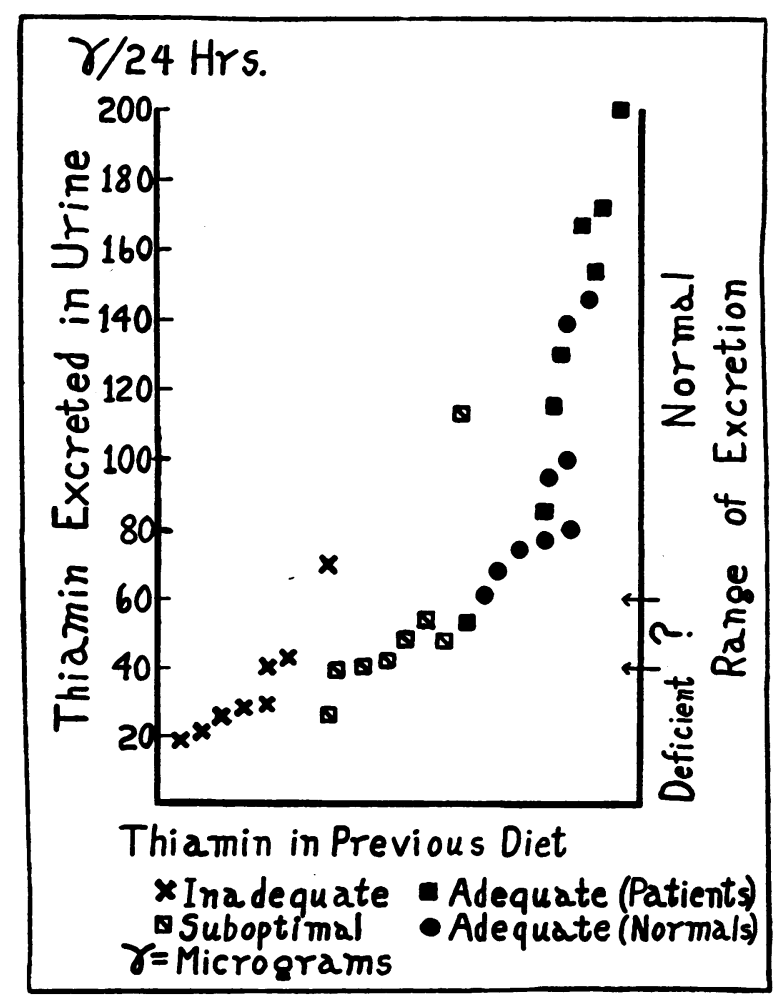

Fig. 2. Correlation in Adult Females Between the Adequacy of the Preceding Dietary Thiamin INTAKE AND THE 24-HOUR URINARY THIAMIN EXCRETION 


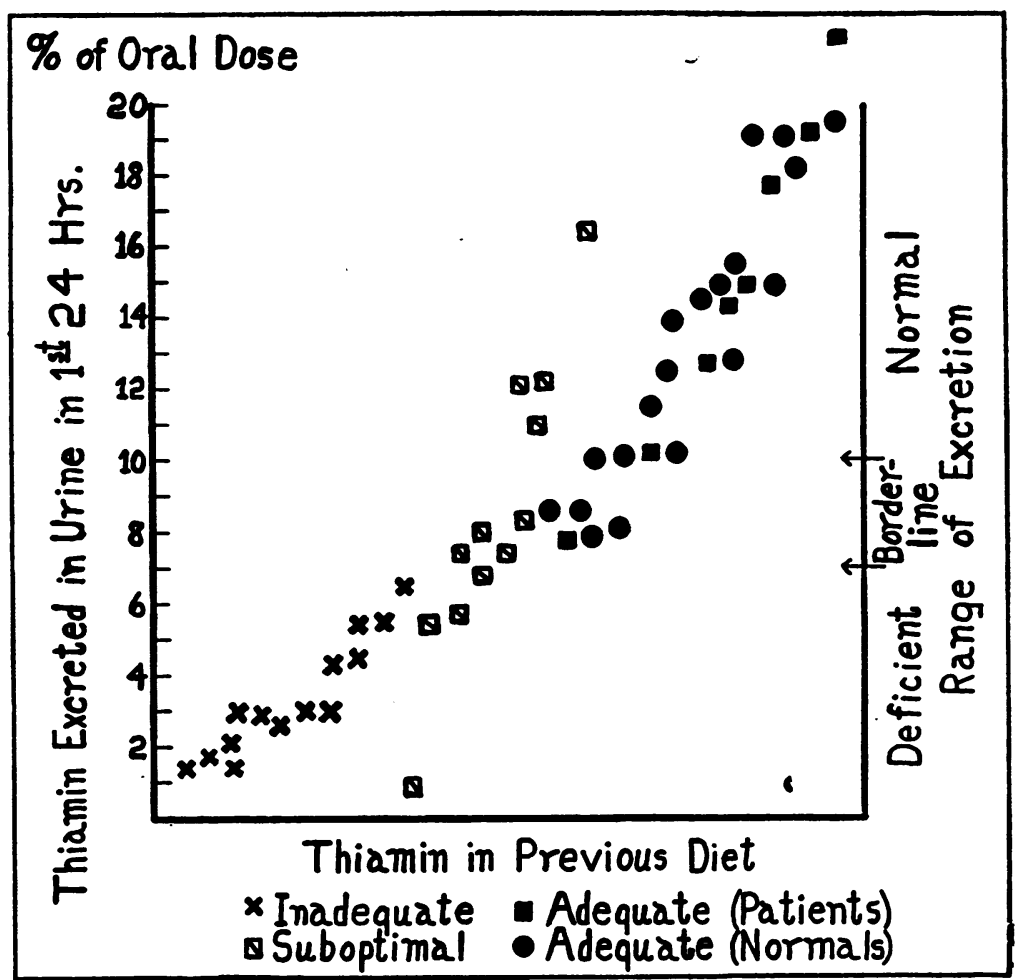

Fig. 3. Correlation in Adult Males and Females Between the Adequacy of the Preceding Dietary Thiamin Intake and the Percentage of a 5 mgm. Test Dose of Thiamin Excreted in the Urine During the First 24 Hours After It Is Taken Orally with a Meal

The excretion values are corrected for the amount of thiamin from dietary sources excreted during the test period.

indicative of a fairly certain depletion. Excretion by a member of either sex of less than 7 per cent of a $5 \mathrm{mgm}$. oral test dose taken with a meal is also evidence of depletion of thiamin stores. It is pertinent to note that values for the normal excretions used in estimating these standards are obtained from subjects in whom no attempt at saturation with the vitamin has been made. These minimal standards for thiamin excretion are purposely conservative.

Patients with clinical thiamin deficiency. Three patients with peripheral polyneuritis, clinically identical with that of beriberi (4), were studied. Case 1 in addition presented all the findings of " beriberi heart disease" (5). Alcoholism was responsible for a grossly inadequate dietary intake in each case. In these patients, the values for thiamin excretion, presented in Table $I$, are much below those of the controls, both before and after the oral dose. Each of these patients also ex-
TABLE I

Urinary excretion of thiamin by patients with clinical thiamin deficiency

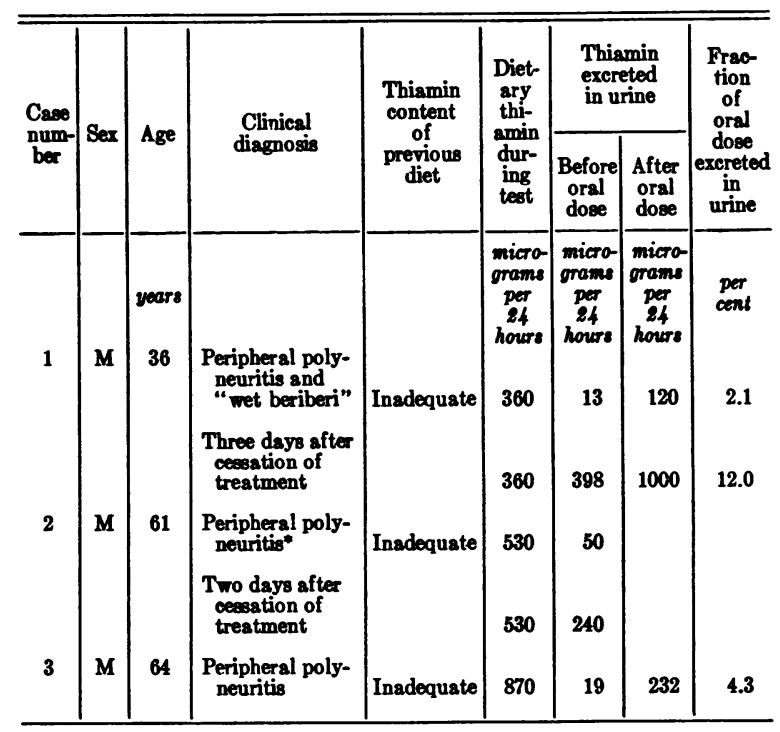

* This patient also had clinical evidence of scurvy, and a plasma ascorbic acid value of $0.17 \mathrm{mgm}$. per $100 \mathrm{cc}$. 
creted definitely less thiamin in the urine after parenteral test doses than did normal subjects. All 3 patients showed excellent clinical improvement after treatment with crystalline thiamin, adequate diets, and other vitamin supplements as indicated by coincident deficiencies.

After treatment, Cases 1 and 2 returned to the diets on which the original tests were done and showed urinary excretions in the high normal range. The high urinary excretion after treatment, when no test dose was given, may be due, in part, to excess storage of the vitamin given therapeutically. Subsequent studies (2) have indicated that a longer interval is required for the urinary thiamin excretion of a normal subject to

TABLE II

Urinary excretion of thiamin by patients with nutritional deficiency

\begin{tabular}{|c|c|c|c|c|c|c|c|c|}
\hline \multirow{2}{*}{$\begin{array}{l}\frac{8}{1} \\
\text { 量 } \\
8 \\
0 \\
0\end{array}$} & \multirow[b]{2}{*}{$\frac{5}{8}$} & \multirow[b]{2}{*}{8} & \multirow{2}{*}{ 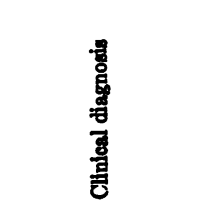 } & \multirow{2}{*}{ 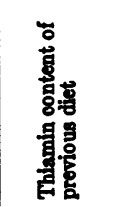 } & \multirow{2}{*}{ 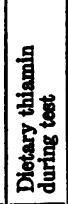 } & \multicolumn{2}{|c|}{$\begin{array}{l}\text { Thiamin } \\
\text { excreted } \\
\text { in urine }\end{array}$} & \multirow{2}{*}{ 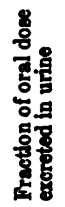 } \\
\hline & & & & & & 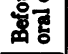 & 安这 & \\
\hline & & years & & & & $\begin{array}{c}\text { micro- } \\
\text { orams } \\
\text { per } \\
\text { 84 } \\
\text { hours }\end{array}$ & $\begin{array}{l}\text { micro- } \\
\text { grames } \\
\text { per } \\
24 \\
\text { hours }\end{array}$ & per \\
\hline $\begin{array}{l}4 \\
5 \\
6 \\
7 \\
8\end{array}$ & $\begin{array}{l}\mathbf{F} \\
\mathbf{F} \\
\mathbf{F} \\
\mathbf{F}\end{array}$ & $\begin{array}{l}40 \\
19 \\
33 \\
53 \\
19\end{array}$ & $\begin{array}{l}\text { Pellagra } \\
\text { Pellagra } \\
\text { Pellagra } \\
\text { Pellagra } \\
\text { Pellagra, and }\end{array}$ & $\begin{array}{l}\text { Adequato } \\
\text { Suboptimal } \\
\text { Suboptimal } \\
\text { Suboptimal } \\
\text { Suboptimal }\end{array}$ & $\begin{array}{l}\text { Good } \\
\text { Fair } \\
\text { Fair } \\
\text { Good } \\
\text { Low }\end{array}$ & $\begin{array}{r}167 \\
29 \\
39 \\
48 \\
42\end{array}$ & $\begin{array}{r}882 \\
1308 \\
858 \\
600 \\
414\end{array}$ & $\begin{array}{r}14.3 \\
25.6 \\
16.4 \\
11.0 \\
7.4\end{array}$ \\
\hline $\begin{array}{r}9 \\
10\end{array}$ & $\mathbf{F}$ & $\begin{array}{l}38 \\
18\end{array}$ & $\begin{array}{l}\text { Pellagra } \\
\text { Pellagra_"anorexis }\end{array}$ & $\begin{array}{l}\text { Suboptimal } \\
\text { Inadequate }\end{array}$ & Fair & $\begin{array}{l}54 \\
43\end{array}$ & 366 & 6.5 \\
\hline 11 & $\mathbf{F}$ & 44 & Pellagra and & Inadequate & Low & 26 & & $\dagger$ \\
\hline $\begin{array}{l}12 \\
13 \\
14\end{array}$ & $\begin{array}{l}\mathbf{F} \\
\mathbf{F}\end{array}$ & $\begin{array}{l}37 \\
34 \\
47\end{array}$ & travidarum & $\begin{array}{l}\text { Inadequate } \\
\text { Inadequate } \\
\text { Inadequate }\end{array}$ & $\begin{array}{l}\text { Good } \\
\text { Low } \\
\text { Fair }\end{array}$ & $\begin{array}{l}28 \\
18 \\
21\end{array}$ & $\begin{array}{l}300 \\
292 \\
170\end{array}$ & $\begin{array}{l}5.4 \\
5.5 \\
3.0\end{array}$ \\
\hline $\begin{array}{l}16 \\
17 \\
18 \\
19\end{array}$ & $\begin{array}{l}\mathbf{M} \\
\mathbf{M} \\
\mathbf{M} \\
\mathbf{M}\end{array}$ & $\begin{array}{l}29 \\
24 \\
61 \\
57\end{array}$ & $\begin{array}{l}\text { Pellagra and mioro- } \\
\text { cytio anemis } \\
\text { After treatment } \\
\text { Angular stomatitis } \\
\text { Hypovitaminosis A* } \\
\text { Pellagra } \\
\text { Pellagra and }\end{array}$ & $\begin{array}{l}\text { Inadequate } \\
\text { Suboptimal } \\
\text { Suboptimal } \\
\text { Suboptimal } \\
\text { Inadequate }\end{array}$ & $\begin{array}{l}\text { Good } \\
\text { Good } \\
\text { Fair } \\
\text { Low } \\
\text { Good } \\
\text { Fair }\end{array}$ & $\begin{array}{r}40 \\
208 \\
62 \\
52 \\
92 \\
37\end{array}$ & $\begin{array}{r}270 \\
1765 \\
666 \\
660 \\
450 \\
1285\end{array}$ & $\begin{array}{r}4.6 \dagger \\
31.1 \ddagger \\
12.1 \\
11.0 \\
7.4 \\
25.0\end{array}$ \\
\hline 20 & $\mathbf{M}$ & 61 & and & Inadequate & Low & 22 & & $t$ \\
\hline 21 & $\mathbf{M}$ & 64 & Id neo- & Inadequate & Good & 32 & 180 & 3.0 \\
\hline 22 & $\mathbf{M}$ & 57 & Pellagra and & Inadequate & Good & 27 & 176 & 3.0 \\
\hline 23 & $\mathbf{M}$ & 54 & Pellagre, scurvy and & Inadequate & Good & 61 & 146 & 1.7 \\
\hline 24 & $\mathbf{M}$ & 58 & $\begin{array}{l}\text { Pellagra and } \\
\text { Hodgtin's disease* }\end{array}$ & Inadequate & Fair & 66 & 138 & 1.4 \\
\hline
\end{tabular}

* These patients had plasma ascorbic acid values of less than $0.5 \mathrm{mgm}$. per $100 \mathrm{cc}$. on or shortly after admission. $\dagger$ These patients also excreted during continuous intravenous administration of thiamin significantly less of the vitamin than did normal controls.

$\ddagger$ After treatment, the excretion of thiamin during continuous intravenous administration was also in the range of the normal control values. return to its basal level after discontinuation of supplementary thiamin.

Patients with other nutritional deficiencies. Twenty-one patients who presented clinical evidence of single or multiple nutritional deficiencies as a major, and often the sole, cause of symptoms were studied. The results are presented in Table II. None of these cases presented unequivocal clinical evidence of thiamin deficiency. It should be noted that we have made the diagnosis of pellagra in patients who have manifestations other than the classical dermatitis, and that many foods with a fair to good thiamin content (3) are not highly pellagra-preventive (6). The clinical diagnosis in these cases was confirmed by observation of a significant response to therapy with appropriate vitamin supplements. All but 1 patient showed excretions before the test dose definitely lower than the values obtained on normals, and 9 of the 18 cases given the oral test dose excreted less than 7 per cent thereof. Cases 5 and 19 show apparently paradoxical values, with low excretion on the diet alone and unusually high values after the oral dose. The tests were repeated on Case 15 8 days after the cessation of treatment with a yeast and liver concentrate of high thiamin content. Values well in the normal range were obtained then.

Patients with organic heart disease. The fact that thiamin deficiency per se can cause serious cardiovascular disturbances $(5,7,8)$ has led to consideration of the possibility that a concomitant but less evident deficiency may contribute to the clinical disability in organic heart disease $(9,10$, 11). Restriction of the intake of the "protective foods" is not uncommon in chronic cardiac disease, either as a result of gastro-intestinal symptoms or as part of the therapeutic regimen. Table III presents the urinary thiamin excretion values of 7 patients who were hospitalized for treatment of cardiac decompensation. There was no evidence of impairment of renal function in these cases. Case 31, the only one to show excretions definitely in the normal range, was tested after recovery from decompensation. However, all 7 patients responded satisfactorily to the usual treatment with bed rest, digitalization and diuretics, on an adequate diet without vitamin supplements. Cases 26, 28, and 29 failed to show any additional 
TABLE III

Urinary excretion of thiamin by patients with organic heart disease

\begin{tabular}{|c|c|c|c|c|c|c|c|c|c|}
\hline \multirow{2}{*}{$\begin{array}{c}\text { Case } \\
\text { number }\end{array}$} & \multirow{2}{*}{ Sex } & \multirow{2}{*}{ Age } & \multirow{2}{*}{$\begin{array}{l}\text { Type of heart } \\
\text { disease }\end{array}$} & \multirow{2}{*}{$\begin{array}{l}\text { Degree of } \\
\text { decompensa- } \\
\text { tion when } \\
\text { tested }\end{array}$} & \multirow{2}{*}{$\begin{array}{l}\text { Thiamin } \\
\text { content of } \\
\text { previous } \\
\text { diet }\end{array}$} & \multirow{2}{*}{$\begin{array}{c}\text { Dietary } \\
\text { thiamin } \\
\text { during } \\
\text { test }\end{array}$} & \multicolumn{2}{|c|}{$\begin{array}{l}\text { Thiamin excreted } \\
\text { in urine }\end{array}$} & \multirow{2}{*}{$\begin{array}{l}\text { Fraction of } \\
\text { oral doee } \\
\text { excreted } \\
\text { in urine }\end{array}$} \\
\hline & & & & & & & $\begin{array}{c}\text { Before } \\
\text { oral dose }\end{array}$ & $\begin{array}{c}\text { After } \\
\text { oral dose }\end{array}$ & \\
\hline & & years & & & & & $\begin{array}{c}\text { micrograms } \\
\text { per } \\
24 \text { hours }\end{array}$ & $\begin{array}{c}\text { micrograms } \\
\text { per } \\
24 \text { hours }\end{array}$ & per cent \\
\hline 25 & $\mathbf{M}$ & 50 & Hypertensive & $+t+t$ & Inadequate & $\begin{array}{l}\text { Fair } \\
(650 \gamma)\end{array}$ & 33 & 180 & 2.9 \\
\hline 26 & $\mathbf{M}$ & 54 & Hypertensive & $++t$ & Suboptimal & $\begin{array}{l}\text { Fair } \\
\left(500_{\gamma}\right)\end{array}$ & 19 & 63 & 0.9 \\
\hline 27 & $\mathbf{M}$ & 54 & Coronary & $+t+$ & Suboptimal & Good & 64 & 348 & 5.7 \\
\hline $\begin{array}{l}28 \\
29 \\
30 \\
31\end{array}$ & $\begin{array}{l}\mathbf{M} \\
\mathbf{M} \\
\mathbf{F} \\
\mathbf{M}\end{array}$ & $\begin{array}{l}66 \\
37 \\
52 \\
43\end{array}$ & $\begin{array}{l}\text { Arteriosclerotic } \\
\text { Rheumatic } \\
\text { Hypertensive } \\
\text { Hypertensive }\end{array}$ & $\begin{array}{l}++ \\
+ \\
+ \\
0\end{array}$ & $\begin{array}{l}\text { Suboptimal } \\
\text { Suboptimal } \\
\text { Suboptimal } \\
\text { Adequate }\end{array}$ & $\begin{array}{l}\text { Fair } \\
\text { Fair } \\
\text { Fair } \\
\text { Good } \\
(700 \gamma)\end{array}$ & $\begin{array}{r}74 \\
59 \\
113 \\
104\end{array}$ & $\begin{array}{l}384 \\
850\end{array}$ & $\begin{array}{r}5.4 \\
14.9\end{array}$ \\
\hline
\end{tabular}

improvement objectively when large doses of crystalline thiamin were given.

Patients with endocrine disorders. By inference from work on experimental animals, some of the diseases of endocrine origin have been suspected of being associated with abnormalities in utilization of thiamin. Himwich and associates (12) have accelerated the appearance of vitamin B deficiency in dogs by massive doses of thyroid.
Other workers $(13,14,15,16)$ have demonstrated that the amount of thiamin required to maintain weight gain in rats and pigeons is increased by experimentally-induced hyperthyroidism. Frazier and Ravdin (17) have reported improved results by the use of yeast and thiamin in the preoperative preparation of hyperthyroid patients. Table IV presents the values for thiamin excretion on 7 patients with toxic goiter, 6 of whom show values

TABLE IV

Urinary excretion of thiamin by patients with endocrine disorders

\begin{tabular}{|c|c|c|c|c|c|c|c|c|}
\hline \multirow{2}{*}{$\begin{array}{c}\text { Case } \\
\text { number }\end{array}$} & \multirow{2}{*}{ Sex } & \multirow{2}{*}{ Age } & \multirow{2}{*}{ Clinical diagnosis } & \multirow{2}{*}{$\begin{array}{l}\text { Thiamin } \\
\text { content of } \\
\text { previous } \\
\text { diet }\end{array}$} & \multirow{2}{*}{$\begin{array}{l}\text { Dietary } \\
\text { thiamin } \\
\text { during } \\
\text { test }\end{array}$} & \multicolumn{2}{|c|}{$\begin{array}{l}\text { Thiamin excreted } \\
\text { in urine }\end{array}$} & \multirow{2}{*}{$\begin{array}{l}\text { Fraction of } \\
\text { oral dose } \\
\text { excreted } \\
\text { in urine }\end{array}$} \\
\hline & & & & & & $\begin{array}{c}\text { Before } \\
\text { oral dose }\end{array}$ & $\begin{array}{c}\text { After } \\
\text { oral dose }\end{array}$ & \\
\hline & & years & & & & $\begin{array}{c}\text { micrograms } \\
\text { per } \\
24 \text { hours }\end{array}$ & $\begin{array}{c}\text { micrograms } \\
\text { per } \\
\text { 84 hours }\end{array}$ & per cent \\
\hline $\begin{array}{l}32 \\
33 \\
34\end{array}$ & $\begin{array}{l}\text { F } \\
\text { F } \\
\text { F }\end{array}$ & $\begin{array}{l}36 \\
47 \\
36\end{array}$ & $\begin{array}{l}\text { Exophthalmic goiter } \\
\text { Exophthalmic goiter } \\
\text { Recurrent exophthalmic } \\
\text { goiter }\end{array}$ & $\begin{array}{l}\text { Adequate } \\
\text { Adequate } \\
\text { Adequate }\end{array}$ & $\begin{array}{l}\text { Good } \\
\text { Good } \\
\text { Good }\end{array}$ & $\begin{array}{r}106 \\
56 \\
157\end{array}$ & $\begin{array}{r}1206 \\
750 \\
750\end{array}$ & $\begin{array}{l}22.0 \\
13.9 \\
11.9\end{array}$ \\
\hline 35 & $\mathbf{M}$ & 35 & $\begin{array}{l}\text { Toxic adenomatous } \\
\text { goiter }\end{array}$ & Adequate & Good & 149 & 1140 & 19.8 \\
\hline $\begin{array}{l}36 \\
37 \\
38\end{array}$ & $\begin{array}{l}\mathbf{M} \\
\mathbf{M} \\
\mathbf{M}\end{array}$ & $\begin{array}{l}34 \\
20 \\
47\end{array}$ & $\begin{array}{l}\text { Exophthalmic goiter } \\
\text { Exophthalmic goiter } \\
\text { Exophthalmic goiter }\end{array}$ & $\begin{array}{l}\text { Adequate } \\
\text { Adequate } \\
\text { Inadequate for } \\
2 \% \text { weeks }\end{array}$ & $\begin{array}{l}\text { Good } \\
\text { Good } \\
\text { Good }(980 \gamma)\end{array}$ & $\begin{array}{r}188 \\
195 \\
44\end{array}$ & $\begin{array}{r}1026 \\
732 \\
182\end{array}$ & $\begin{array}{r}16.8 \\
10.7 \\
2.8\end{array}$ \\
\hline $\begin{array}{l}39 \\
40 \\
41 \\
42 \\
43 \\
44 \\
45\end{array}$ & $\begin{array}{l}\mathbf{F} \\
\mathbf{F} \\
\mathbf{F} \\
\mathbf{F} \\
\mathbf{M} \\
\mathbf{M} \\
\mathbf{M}\end{array}$ & $\begin{array}{l}51 \\
52 \\
53 \\
67 \\
68 \\
25 \\
27\end{array}$ & $\begin{array}{l}\text { Myxedema } \\
\text { Diabetes mellitus* } \\
\text { Diabetes mellitus* } \\
\text { Diabetes mellitust } \\
\text { Diabetes mellitust } \\
\text { Diabetes mellitus } \\
\text { Diabetes mellitusf }\end{array}$ & $\begin{array}{l}\text { Suboptimal } \\
\text { Adequate } \\
\text { Adequate } \\
\text { Adequate } \\
\text { Adequate } \\
\text { Adequate } \\
\text { Adequate }\end{array}$ & $\begin{array}{l}\text { Fair } \\
\text { Good }(900 \gamma) \\
\text { Good } \\
\text { Good } \\
\text { Good } \\
\text { Fair } \\
\text { Good }\end{array}$ & $\begin{array}{r}18 \\
70 \\
144 \\
46 \\
127 \\
103 \\
300\end{array}$ & $\begin{array}{r}230 \\
705 \\
846 \\
924 \\
690 \\
1152\end{array}$ & $\begin{array}{r}4.2 \\
12.7 \\
14.0 \\
17.6 \\
11.7 \\
17.0\end{array}$ \\
\hline
\end{tabular}

* No neurologic complaints; neurologic examination normal.

+ Symptoms, sensory disturbances and reflex changes of peripheral neuritis.

$¥$ No symptoms or sensory disturbances. Absent tendon reflexes in lower extremities. 
well within the normal range. These patients had experienced the usual polyphagia of their disease and had taken diets of high caloric and vitamin content. Case 38, who showed definitely low urinary thiamin values, had also eaten well until anorexia developed about 18 days prior to the tests. He was extremely toxic on admission and went into thyroid crisis which terminated fatally on the seventh day of hospitalization. These findings suggest that in hyperthyroidism the usual increase in food consumption suffices to meet the increased requirement for thiamin, but that in the occasional patient with poor appetite or gastrointestinal disturbances a deficiency of this vitamin may rapidly develop. The 1 case with classical myxedema studied showed low values for thiamin excretion; this patient had not eaten well prior to the test.

The steadily mounting experimental evidence that thiamin plays a rôle in the intermediary metabolism of carbohydrates (18) has aroused considerable interest in the status of diabetics with regard to this vitamin (19). Of particular interest is the relationship of "diabetic neuritis" to the peripheral neuritis of beriberi. All of 6 regulated diabetics studied showed excretory values in the normal range. These patients had all been on diets of adequate thiamin content before coming

TABLE V

Urinary excretion of thiamin by patients with gastro-intestinal disease

\begin{tabular}{|c|c|c|c|c|c|c|c|c|c|c|}
\hline \multirow{2}{*}{$\begin{array}{c}\text { Case } \\
\text { num- } \\
\text { ber }\end{array}$} & \multirow{2}{*}{ Sex } & \multirow{2}{*}{ Age } & \multirow{2}{*}{ Diagnosis } & \multirow{2}{*}{$\begin{array}{l}\text { Thiamin } \\
\text { content of } \\
\text { previous } \\
\text { diet }\end{array}$} & \multirow{2}{*}{$\begin{array}{c}\text { Dietary } \\
\text { thiamin } \\
\text { during } \\
\text { test }\end{array}$} & \multirow{2}{*}{$\begin{array}{l}\text { Antacid medication } \\
\text { during test }\end{array}$} & \multicolumn{2}{|c|}{$\begin{array}{l}\text { Thiamin } \\
\text { excreted } \\
\text { in urine }\end{array}$} & \multirow{2}{*}{$\mid \begin{array}{c}\text { Frac- } \\
\text { tion } \\
\text { of } \\
\text { oral } \\
\text { dose } \\
\text { in } \\
\text { urine }\end{array}$} & \multirow{2}{*}{ Comment } \\
\hline & & & & & & & $\begin{array}{c}\text { Before } \\
\text { oral } \\
\text { dose }\end{array}$ & $\begin{array}{l}\text { After } \\
\text { oral } \\
\text { dose }\end{array}$ & & \\
\hline & & years & & & & & $\begin{array}{c}\text { micro- } \\
\text { grams } \\
\text { per } \\
24 \\
\text { hours }\end{array}$ & $\begin{array}{c}\text { micro- } \\
\text { grams } \\
\text { per } \\
\text { \&4 } \\
\text { hours }\end{array}$ & $\begin{array}{l}\text { per } \\
\text { cent }\end{array}$ & \\
\hline 46 & $\mathbf{M}$ & 39 & $\underset{\text { ulcer }}{\text { Marginal }}$ & Adequate & Good & $\begin{array}{l}\mathrm{CaCO}_{8} 1 \text { gram and } \\
\mathrm{Mg}_{2} \mathrm{Si}_{3} \mathrm{O}_{8} 0.5 \text { grams }\end{array}$ & 99 & 792 & 13.9 & $\begin{array}{l}2 \text { years after gastro- } \\
\text { enterostomy }\end{array}$ \\
\hline 47 & $\mathbf{M}$ & 25 & $\begin{array}{l}\text { Duodenal } \\
\text { ulcer }\end{array}$ & Adequate & Good & $\mathrm{CaCO}_{3} 2$ grams or & 34 & 720 & 13.7 & $\begin{array}{l}\text { Recent chronic hem- } \\
\text { orrhage }\end{array}$ \\
\hline 48 & $\mathbf{M}$ & 34 & $\begin{array}{l}\text { Duodenal } \\
\text { ulcer }\end{array}$ & Adequate & Good & $\mathrm{CaCO}_{3} 2$ grams or & 15 & 630 & 12.3 & $\begin{array}{l}\text { Recent chronic hem- } \\
\text { orrhage }\end{array}$ \\
\hline 49 & $\mathbf{M}$ & 58 & Atrophic & Adequate & Good & Colloidal $\mathrm{Al}(\mathrm{OH})_{3}$ & 41 & 625 & 11.7 & 8 days after large \\
\hline 50 & $\mathbf{M}$ & 29 & $\begin{array}{l}\text { Duodenal } \\
\text { ulcer }\end{array}$ & Adequate & Good & $\begin{array}{c}\mathrm{CaCO}_{3} 2 \text { grams or } \\
\mathrm{MgCO}_{3} 2 \text { grams }\end{array}$ & 32 & & & \\
\hline 51 & $\mathbf{M}$ & 56 & $\begin{array}{l}\text { Gastric } \\
\text { ulcer }\end{array}$ & Adequate & Good & $\begin{array}{l}\mathrm{qa} \cdot \mathrm{CO}_{3} 2 \text { grams or } \\
\mathrm{MgCO}_{3} 2 \text { grams } \\
\text { q. } 1 \text { hour }\end{array}$ & 31 & & & $\begin{array}{l}\text { X-ray showed ulcer } \\
\text { healed at time of } \\
\text { test }\end{array}$ \\
\hline 52 & $\mathbf{M}$ & 66 & $\begin{array}{l}\text { Duodenal } \\
\text { ulcer }\end{array}$ & Adequate & Good & $\begin{array}{l}\mathrm{CaCO}_{2} 2 \text { grams or } \\
\mathrm{MgCO}_{8} 2 \text { grams } \\
\text { q. } 1 \text { hour }\end{array}$ & 63 & 594 & 10.6 & $\begin{array}{l}\text { Mild chronic pyloric } \\
\text { obstruction }\end{array}$ \\
\hline 53 & $\mathbf{M}$ & 65 & $\begin{array}{l}\text { Duodenal } \\
\text { ulcer }\end{array}$ & Adequate & Good & $\begin{array}{l}\text { Colloidal } \mathrm{Al}(\mathrm{OH})_{3} \\
6 \text { cc. q. } 1 \text { hour }\end{array}$ & 30 & 492 & 9.2 & $\begin{array}{l}\text { Recent chronic hem- } \\
\text { orrhage }\end{array}$ \\
\hline 54 & $\mathbf{M}$ & 58 & $\begin{array}{l}\text { Duodenal } \\
\text { ulcer }\end{array}$ & Adequate & Good & $\begin{array}{l}\mathrm{CaCO}_{3} 2 \text { grams q. } \\
\text { 2.hours }\end{array}$ & 46 & & & \\
\hline 55 & $\mathbf{M}$ & 49 & $\begin{array}{l}\text { Duodenal } \\
\text { ulcer }\end{array}$ & Adequate & Good & $\begin{array}{l}\mathrm{CaCO}_{8} 2 \text { grams or } \\
\mathrm{MgCO}_{3} 2 \text { mgm. q. } \\
1 \text { hour }\end{array}$ & 21 & 428 & 8.1 & $\begin{array}{l}\text { Just after relief of } \\
\text { partial obstruction }\end{array}$ \\
\hline 56 & $\mathbf{M}$ & 56 & $\begin{array}{c}\text { Recurrent } \\
\text { marginal }\end{array}$ & Adequate & Good & $\begin{array}{l}\text { Colloidal } \mathrm{Al}(\mathrm{OH})_{3} \\
4 \text { cc. q. } 1 \text { hour }\end{array}$ & 43 & 373 & 6.6 & $\begin{array}{l}19 \text { years after gas- } \\
\text { tro-enterostomy }\end{array}$ \\
\hline 57 & $\mathbf{M}$ & 56 & $\begin{array}{l}\text { ulcer } \\
\text { Duodenal } \\
\text { ulcer }\end{array}$ & Suboptimal & Good & $\begin{array}{l}\text { Colloidal } \mathrm{Al}(\mathrm{OH})_{3} \\
8 \text { cc. q. } 1 \text { hour }\end{array}$ & 38 & 366 & 6.6 & $\begin{array}{l}\text { Recurrent hemor- } \\
\text { rhages for } 10 \text { years }\end{array}$ \\
\hline 58 & $\mathbf{M}$ & 47 & $\begin{array}{l}\text { Duodenal } \\
\text { ulcer }\end{array}$ & Adequate & Good & $\begin{array}{l}\text { Colloidal } \mathrm{Al}(\mathrm{OH})_{3} \\
6 \text { cc. q. } 1 \text { hour }\end{array}$ & 33 & 258 & 4.5 & $\begin{array}{l}\text { Tests on } 4 \text { th and } 5 \text { th } \\
\text { hospital day }\end{array}$ \\
\hline 59 & $\mathbf{M}$ & 46 & $\begin{array}{l}\text { Duodenal } \\
\text { ulcer }\end{array}$ & Suboptimal & Good & $\begin{array}{l}\text { Colloidal } \mathrm{Al}(\mathrm{OH})_{3} \\
6 \text { cc. q. } 2 \text { hours }\end{array}$ & 26 & 123 & 1.9 & $\begin{array}{l}4 \text { days after recov- } \\
\text { ery from alkalosis }\end{array}$ \\
\hline
\end{tabular}


TABLE v-Continued

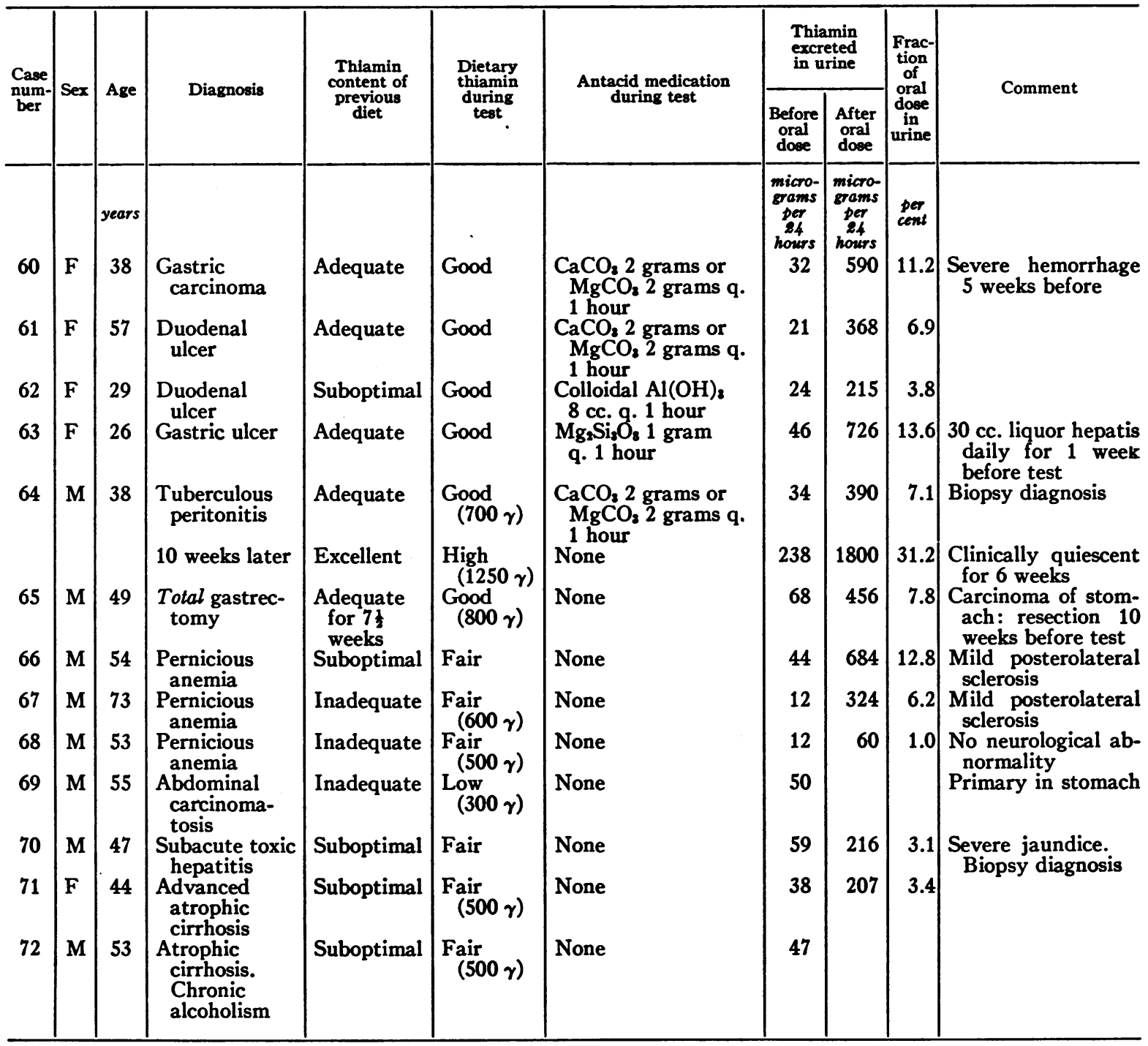

to the hospital and none of them showed evidence of ketosis, although 2 showed intermittent glycosuria and hyperglycemia. Balance studies with more careful attention to the state of control of the diabetes are expected to give more conclusive information.

Patients with gastro-intestinal disease. Nineteen cases were studied while being treated by a modified Sippy regimen for peptic ulcer; subsequently 3 of these were shown to have atrophic gastritis, tuberculous peritonitis, and gastric carcinoma respectively, rather than ulcer. These tests, with one exception, were done after the patients had been under treatment for 2 or more weeks.
Most of the patients had been on good diets before hospitalization. Preceding and during the tests they ate their diets well. Calculations of these diets showed a daily intake of 700 to 900 micrograms of thiamin with a thiamin-non-fatcalorie ratio of approximately 1.0. Antacid medication was continued during the test period.

The results are presented in Table V. The values before the oral dose are definitely lower than those obtained from normal subjects except for 1 case; however, only 5 of the 16 given oral test doses excreted less than 7 per cent thereof. This type of response, a low value during the period when the sole source of thiamin is the diet 
followed by excretion of a normal fraction of the oral dose, was observed in normally nourished subjects after subsisting for a few days on an experimentally deficient diet (2). Since the patients on the modified Sippy regimen are known to have ingested an adequate diet during the test, it is suggested that the antacid medication may be the factor responsible for the low urinary thiamin values. This may be due either to destruction of the dietary thiamin in the gastro-intestinal tract when alkali is given simultaneously, or to loss of the vitamin by way of the feces due to adsorption of the alumina gels or silicates. The results in Case 63 support this concept: despite the fact that this patient had received whole vitamin B complex supplements for 1 week prior to the test, the excretion before the oral dose was only 46 micrograms, followed by a percentage excretion of the oral dose well in the normal range. Studies reported previously (1) have ruled out the possi- bility that these results are due to destruction of thiamin by alkaline urine in the bladder.

A somewhat comparable situation might be anticipated in patients with achlorhydria. Case 65 was studied 10 weeks after a total gastric resection for carcinoma; he had ingested and retained a diet of adequate thiamin content for over 7 weeks prior to the test. He excreted less dietary thiamin than did the normal subjects, but the fraction of the oral dose excreted was not definitely low. One of 3 patients with pernicious anemia had values very similar to those patients receiving antacid medication, while the other 2 showed low values both before and after the oral dose.

The low excretory values obtained on the 3 cases of hepatic disease studied are of interest in view of the widely held opinion that the liver has an important rôle in the handling of thiamin (18). However, the results in these cases may be attributed to the preceding suboptimal thiamin in-

TABLE VI

Urinary excretion of thiamin by patients with various other diseases

\begin{tabular}{|c|c|c|c|c|c|c|c|c|c|}
\hline \multirow{2}{*}{$\begin{array}{l}\text { Case } \\
\text { num- } \\
\text { ber }\end{array}$} & \multirow{2}{*}{ Sex } & \multirow{2}{*}{ Age } & \multirow{2}{*}{ Clinical diagnosis } & \multirow{2}{*}{$\begin{array}{l}\text { Thiamin } \\
\text { content of } \\
\text { previous } \\
\text { diet }\end{array}$} & \multirow{2}{*}{$\begin{array}{c}\text { Dietary } \\
\text { thiamin } \\
\text { during } \\
\text { test }\end{array}$} & \multicolumn{2}{|c|}{$\begin{array}{l}\text { Thiamin excreted } \\
\text { in urine }\end{array}$} & \multirow{2}{*}{$\begin{array}{l}\text { Fraction } \\
\text { of dose } \\
\text { excreted } \\
\text { in urine }\end{array}$} & \multirow{2}{*}{ Comment } \\
\hline & & & & & & $\begin{array}{l}\text { Before } \\
\text { oral } \\
\text { dose }\end{array}$ & $\begin{array}{l}\text { After } \\
\text { oral } \\
\text { dose }\end{array}$ & & \\
\hline & & years & & & & $\begin{array}{l}\text { micro- } \\
\text { grams } \\
\text { per } \\
24 \text { hours }\end{array}$ & $\begin{array}{l}\text { micro- } \\
\text { grams } \\
\text { per } \\
24 \text { hours }\end{array}$ & per cent & \\
\hline 73 & $\mathbf{M}$ & 45 & Brucellosis & Inadequate & $\begin{array}{l}\text { Good } \\
(700 \gamma)\end{array}$ & 47 & 176 & 2.6 & $\begin{array}{l}\text { Mild peripheral neu- } \\
\text { ritis }\end{array}$ \\
\hline 74 & $\mathbf{M}$ & 62 & $\begin{array}{l}\text { Carcinoma and osteo- } \\
\text { myelitis of mandible }\end{array}$ & Inadequate & Fair & 38 & & & \\
\hline $\begin{array}{l}75 \\
76\end{array}$ & $\begin{array}{l}\mathbf{M} \\
\mathbf{M}\end{array}$ & $\begin{array}{l}55 \\
48\end{array}$ & $\begin{array}{l}\text { Chronic alcoholism } \\
\text { Myalgia }\end{array}$ & $\begin{array}{l}\text { Adequate } \\
\text { Suboptimal }\end{array}$ & $\begin{array}{l}\text { Good } \\
\text { Fair } \\
\quad(550 \gamma)\end{array}$ & $\begin{array}{l}96 \\
43\end{array}$ & 460 & 8.3 & \\
\hline $\begin{array}{l}77 \\
78\end{array}$ & $\begin{array}{l}\mathbf{M} \\
\mathbf{M}\end{array}$ & $\begin{array}{l}46 \\
52\end{array}$ & $\begin{array}{l}\text { Impotence } \\
\text { Progressive peripheral } \\
\text { polyneuritis }\end{array}$ & $\begin{array}{l}\text { Adequate } \\
\text { Adequate }\end{array}$ & Good & $\begin{array}{l}218 \\
208\end{array}$ & $\begin{array}{r}855 \\
1386\end{array}$ & $\begin{array}{l}12.7 \\
23.6\end{array}$ & $\begin{array}{l}\text { Unaffected by thi- } \\
\text { amin therapy }\end{array}$ \\
\hline 79 & $\mathbf{M}$ & 55 & $\begin{array}{l}\text { Chronic glomerulo- } \\
\text { tubular nephritis with }\end{array}$ & Adequate & $\begin{array}{l}\text { Good } \\
(700 \gamma)\end{array}$ & 23 & 165 & 2.8 & $\begin{array}{l}\text { N.P.N. } 100 \text { to } 75 \\
\text { mgm. per cent }\end{array}$ \\
\hline 80 & $\mathbf{F}$ & 36 & $\begin{array}{l}\text { Chronic glomerulo- } \\
\text { tubular nephritis }\end{array}$ & Suboptimal & Fair & 43 & 445 & 8.0 & N.P.N. normal \\
\hline 81 & $\mathbf{F}$ & 23 & Neurocirculatory & Suboptimal & Fair & 26 & 384 & 7.2 & \\
\hline 82 & $\mathbf{F}$ & 19 & Hysteria & Suboptimal & $\begin{array}{l}\text { Good } \\
(700 \gamma)\end{array}$ & 46 & 435 & 7.8 & \\
\hline $\begin{array}{l}83 \\
84\end{array}$ & $\underset{\mathbf{F}}{\mathbf{F}}$ & $\begin{array}{l}42 \\
55\end{array}$ & $\begin{array}{l}\text { Sciatic neuralgia } \\
\text { Bronchopneumonia }\end{array}$ & $\begin{array}{l}\text { Adequate } \\
\text { Adequate }\end{array}$ & Good & $\begin{array}{r}53 \\
115\end{array}$ & $\begin{array}{r}536 \\
1002\end{array}$ & $\begin{array}{r}9.7 \\
17.7\end{array}$ & $\begin{array}{l}\text { Tested during con- } \\
\text { valescence }\end{array}$ \\
\hline $\begin{array}{l}85 \\
86 \\
87 \\
88 \\
89\end{array}$ & $\begin{array}{l}\mathbf{F} \\
\mathbf{F} \\
\mathbf{F} \\
\mathbf{F} \\
\mathbf{F}\end{array}$ & $\begin{array}{l}20 \\
45 \\
40 \\
28 \\
23\end{array}$ & $\begin{array}{l}\text { Gastro-intestinal allergy } \\
\text { Periarthritis shoulder } \\
\text { Migraine } \\
\text { ? retrobulbar neuritis } \\
\text { Anxiety state }\end{array}$ & $\begin{array}{l}\text { Adequate } \\
\text { Adequate } \\
\text { Adequate } \\
\text { Adequate } \\
\text { Adequate }\end{array}$ & $\begin{array}{l}\text { Fair } \\
\text { Good } \\
\text { Good } \\
\text { Good } \\
\text { Good }\end{array}$ & $\begin{array}{r}85 \\
154 \\
172 \\
130 \\
200\end{array}$ & 1090 & 19.2 & \\
\hline
\end{tabular}


take as well as to an impairment of storage in the body.

Patients with miscellaneous diseases. The results obtained on 17 patients with various other diseases are presented in Table VI. The low values for thiamin excretion by Case 73 suggest that the mild peripheral neuritis which complicated his brucellosis might well be attributed to a thiamin deficiency; however, it was impossible to carry out therapy suitably controlled to rule out a toxic etiology. Case 79 with terminal nephritis and chronic nitrogen retention had eaten an adequate diet for 4 weeks before the test; the low urine values obtained may indicate that in renal insufficiency the kidney is unable to excrete thiamin. Among the other cases in this group there is apparent some correlation between the thiamin content of the preceding diet and the values for urinary thiamin excretion.

\section{DISCUSSION}

The correlation in the individual subject between the urinary excretion of thiamin before and after the oral test dose is concordant when judged by the above standards in 68 of the 75 cases in which both values were obtained, excluding patients with achlorhydria and those receiving antacid medication. We have observed only 1 subject (Case 30, Table III) in whom a normal value for the 24-hour excretion was followed by excretion of an abnormally small fraction of the test dose. The remaining 6 subjects excreted a normal fraction of the test dose despite a preceding 24-hour value in the definitely low range; 4 of these were known to have eaten poorly on the day of the test. These results are interpreted as indicating that insufficient thiamin from dietary sources was available for absorption from the gastro-intestinal tract on the day of the test, but that there was no significant depletion of the thiamin stores. Experimental evidence for this interpretation is presented elsewhere (2).

The results in the patients with achlorhydria and those receiving antacid medication suggest that in these cases diagnostic significance should be attached only to the fraction of the test dose excreted. They also indicate that factors in the gastro-intestinal tract which affect the availability of thiamin for absorption may be of clinical sig- nificance. Preliminary studies indicate that a significant reduction in urinary thiamin excretion follows the administration of antacid medications to patients on a constant dietary intake. Results of preliminary studies on the stability of thiamin in vitro in the secretions of the gastro-intestinal tract indicate that the vitamin is stable in achlorhydric and in normal gastric juice from its natural acidity to $\mathrm{pH}$ of 8 . However, significant losses occur in pancreatic juice and bile at the natural $\mathrm{pH}$ of 8 , but not at $\mathrm{pH} 4.5$. It is suggested that with gastric acidity neutralized or absent the contents of the small intestine may become alkaline enough to permit an abnormal destruction of thiamin.

In discussing standards of normality and deficiency, it is important to bear in mind that low values for urinary thiamin can in themselves indicate no more than a depletion of the body stores of this particular food factor. From the data presented in the tables it is obvious that there is no particular level of urinary thiamin excretion at which signs and symptoms of thiamin deficiency appear. The clinical significance of the finding of thiamin subnutrition in a patient who presents none of the recognized clinical features of thiamin deficiency is uncertain. Before the physiologic alterations and clinical symptoms associated with the wide variety of diseases presented in this paper can be attributed to thiamin deficiency, it will be necessary to evaluate the response of such cases to thiamin therapy under carefully controlled conditions, or to demonstrate a specific biochemical dysfunction.

\section{SUMMARY AND CONCLUSIONS}

Under proper conditions, the level of the urinary excretion of thiamin permits an objective determination of the state of thiamin nutrition in the human subject.

There is good correlation between the urinary thiamin values and the adequacy of the preceding diet with respect to this vitamin. The 24-hour urinary thiamin excretion in subjects whose previous dietary intake of thiamin had been adequate, and who ingested an adequate diet on the day of the test, was 90 micrograms or more in all males, and above 60 micrograms in all females but one. Under similar conditions, the 24-hour excretion in 
subjects whose previous diet had been definitely inadequate in thiamin was 66 micrograms or below in all males, and 43 micrograms or below in all females except one. All subjects with a preceding adequate thiamin intake excreted over 7.5 per cent of an oral test dose of $5 \mathrm{mgm}$. of thiamin given with the largest meal of the day. All subjects whose previous diets had been definitely inadequate excreted less than 7 per cent of such a test dose.

Three patients with "alcoholic beriberi" excreted abnormally small amounts of thiamin in the urine.

About half the patients presenting clinical evidence of deficiencies in other food factors had urinary values indicative of thiamin subnutrition.

Six of the 7 patients with cardiac decompensation due to organic heart disease had values indicative of thiamin subnutrition.

Six patients with regulated diabetes mellitus had urinary thiamin excretions in the normal range.

Six of 7 patients with hyperthyroidism had urinary thiamin values in the normal range. The 1 patient in this group with definitely low values had been on an inadequate thiamin intake for a relatively short time.

Patients receiving antacid medication for peptic ulcer and patients with achlorhydria excreted abnormally small amounts of thiamin in the urine when the diet furnished the entire source of the vitamin. However, most of these patients excreted a normal fraction of the oral test dose.

The authors are indebted to Dr. Walter D. Block for carrying out the plasma ascorbic acid determinations according to the method of Farmer and Abt (20).

\section{BIBLIOGRAPHY}

1. Melnick, D., and Field, H., Jr., Chemical determination, stability, and form of thiamine in urine. $J$. Biol. Chem., 1939, 130, 97.

2. Melnick, D., Field, H., Jr., and Robinson, W. D., A quantitative chemical study of the urinary excretion of thiamine by normal individuals. J. Nutrition, 1939, 18, 593.

3. Williams, R. R., and Spies, T. D., Vitamin $B_{1}$ (Thiamin) and its use in medicine. Macmillan, New York, 1938.

4. Strauss, M. B., The therapeutic use of vitamin $B_{1}$ in polyneuritis and cardiovascular conditions; clinical indications. J. A. M. A., 1938, 110953.

5. Weiss, S., and Wilkins, R. W., The nature of the cardiovascular disturbances in nutritional deficiency states (beriberi). Ann. Int. Med., 1937, 11, 104.

6. Sebrell, W. H., Table showing the pellagra-preventive value of various foods. Pub. Health Rep., 1934, 49, 754.

7. Wenckebach, K. F., Riddle of the beriberi heart. In contributions to the medical sciences in honor of Dr. Emanuel Libman. The International Press, New York, 1932, 3, 1199.

8. Goodhart, R., and Jolliffe, N., The rôle of nutritional deficiencies in the production of cardiovascular disturbances in the alcoholic addict. Am. Heart J., 1938, 15, 569.

9. Jones, W. A., and Sure B., The rôle of vitamin $B_{1}$ in cardiovascular disease; preliminary report. J. Lab. and Clin. Med., 1937, 22, 991.

10. Porter, W. B., Nutritional deficiencies and their relation to the clinical course of heart disease. Virginia M. Monthly, 1938, 65, 459.

11. Bickel, G., Le rôle de l'hypovitaminose $B_{1}$ en pathologie cardio-vasculaire; les myocardies par carence. Presse Méd., 1938, 46, 1916.

12. Himwich, H. E., Goldfarb, W., and Cowgill, G. R., Studies in the physiology of vitamins. XVII. The effect of thyroid administration upon the anorexia characteristic of lack of undifferentiated vitamin B. Am. J. Physiol., 1932, 99, 687.

13. Cowgill, G. R., and Palmieri, M. L., Studies in the physiology of vitamins. XXII. The effect of experimentally induced hyperthyroidism on the vitamin B requirement of pigeons. Am. J. Physiol., 1933, 105, 146.

14. Sure, B., and Smith, M. E., Hyperthyroidism and nutrition; vitamin B and thyroxin. J. Nutrition, 1934, $7,547$.

15. Sure, B., and Buchanan, K. S., Antithyrogenic action of crystalline vitamin $B_{1}$. J. Nutrition, 1937, 13, 513.

16. Drill, V. A., and Sherwood, C. R., The effect of vitamin $B_{1}$ and the vitamin $B_{2}$ complex on the weight, food intake, and estrual cycle of hyperthyroid rats. Am. J. Physiol., 1938, 124, 683.

17. Frazier, W. D., and Ravdin, I. S., The use of vitamin $B_{1}$ in the preoperative preparation of the hyperthyroid patient. Surgery, 1938, 4, 680.

18. Cowgill, G. R., The physiology of vitamin $B_{1}$. J. A. M. A., 1938, 110, 805.

19. Vorhaus, M. G., Williams, R. R., and Waterman, R. E., Studies on crystalline vitamin $B_{1}$ : observations in diabetes. Am. J. Digest. Dis. and Nutrition, 1935, 2, 541.

20. Farmer, C. J., and Abt, A. F., Determination of ascorbic acid content in blood. Proc. Soc. Exper. Med. and Biol., 1935, 32, 1625. 